\title{
HSV Values and OpenCV for Object Tracking
}

\author{
Mir Mahpara Gulzar', Ravinder Pal Singh², and Dr Monika Mehra ${ }^{3}$ \\ ${ }^{1}$ M.Tech, Electronics and Communication Engineering, RIMT University, Punjab, India \\ ${ }^{2}$ Technical Head, Department of Research, Innovations and Incubation, RIMT University, Punjab, India \\ ${ }^{3}$ Head of Department, Electronics and Communication Engineering, RIMT University, Punjab, India \\ Correspondence should be addressed to Mir Mahpara Gulzar; meermahie12@gmail.com
}

Copyright (C) 2022 Mir Mahpara Gulzar et al. This is an open-access article distributed under the Creative Commons Attribution License, which permits unrestricted use, distribution, and reproduction in any medium, provided the original work is properly cited.

\begin{abstract}
This research shows how colour and motion may be utilised to speed up the surveillance of things. Video tracing is a technique for detecting a huge vehicle over a long distance using a camera. The main goal of video tracking is to link objects in subsequent video frames. When objects move faster than the frames per second, maintaining connection might be difficult. Using Hue saturation space values and OpenCV in separate video frames, this article shows how to follow moving objects in real-time. We begin by finding the HSV value of the object to be tested, and then we understand the steps along. The tracking of the items was shown to be 90 percent accurate.
\end{abstract}

KEYWORDS- HSV, OpenCV, Object tracking, Video frames, GUI

\section{INTRODUCTION}

Observing is the act of standing back and watching people's actions and/or activities. Security cameras are the most commonly used gadget. Industrial processes, traffic, and reduced crime are all monitored by these cameras. Despite their widespread use, security cameras have a number of drawbacks. Because these cameras are fixed on motorized hinges, they can only monitor from certain angles [1], and the security system can only be penetrated through hidden regions. The presence of human operators [2,] who are responsible for checking several camera inputs is one source of worry. Criminal or other undesirable behaviors may go unchecked since such operators are subjected to tedium, tiredness, and attention.. As a result, a mobile robot might be used to address these issues. A robotic might freely and continuously tour the surveillance zones, making its own decisions and recognizing unwanted behaviors or activities, then reacting as needed, for as by sending out messages..

Computer vision is significantly used in robotic surveillance to track things. Object tracking is a technique for keeping track of moving objects in a video feed. This can be accomplished by identifying and analyzing a certain attribute, such as the color of a moving item. After that, the technique may be used to monitor the object's progress over time. The vast majority of color tracking systems now available are only designed to track a single color. Due to the shifting circumstances, the tracking color feature may not be as visible as it should be as the camera travels. In this case, tracking might be used to keep an eye on a false object. As a result, new data-driven approaches for predicting the color function have evolved...

\section{A. Hue Saturation and Value (HSV):}

Compared to RGB and CMYK, HSV is more closely linked to human color perception. It has three components: hue, saturation, and value. The color space stores colors and optimization (tones or hues) (saturation or quantity of grey). Deny the reality that HSV and HSB seem to be the same, certain color analyzers, such as those in Adobe Photoshop, utilize the HSB acronym, which substitutes "value" for "brightness." The HSV color wheel might have the form of a cone or a cylinder as shown in Fig 1, but it always has three sections:

Hue is a measure that describes the color part of the framework and runs from 0 to 360 degrees.

The color red has a temperature difference of $0^{\circ} \mathrm{C}$ to $60^{\circ} \mathrm{C}$. Yellow emerges between 61 and 120 degrees.

The color green fades from 121 to 180 degrees.

The temp for cyan is 181 to 240 degrees Fahrenheit.

The temp of blue is 241 to 300 degrees Fahrenheit.

Magenta has a temperature range of 301 to $360^{\circ} \mathrm{C} \ldots$

Saturation: Saturation refers to the amount of grey in a colour that fluctuates between 0 and 100 percent. When this component is set to 0 , the image appears darker and faded. Intensity ranges from 0 to 1 , with 0 representing grey and 1 representing the dominant colour.

Value (brightness): On a scale of 0 to 100 percent, value represents the intensity or colour depth of a colour, with 0 being completely black and 100 being the greatest and most vibrant. 


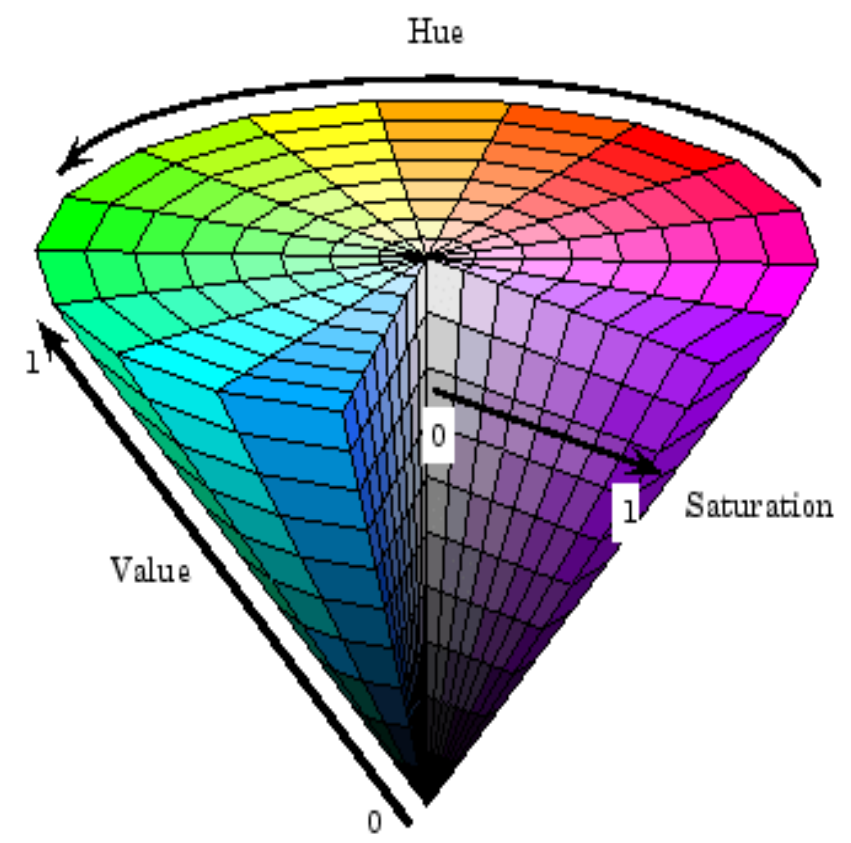

Fig. 1: HSV Cylindrical Cone

\section{B. OpenCV}

OpenCV is a set of software tools mostly used for real-time machine learning. In basic terms, the toolkit is used for picture analysis. It's mostly used for editing photos. This approach might be used to recognize objects, people, or even human handwriting in images and videos. Python can handle the OpenCV analysis array structure when used in conjunction with several programs like as Numpy. To recognize visual patterns and their many features, we use vector space and arithmetic operations on these attributes..

\section{PROBLEM STATEMENT}

Monitoring means keeping an eye on someone's decisions and/or behavior from afar. Security cameras are the most commonly used equipment. Production lines, transit, and criminal security are just a few of the applications for these cameras. Despite their widespread use, security cameras have severe limitations. Because the lens is mounted to metal hinges, it can only view at specific angles, and the safety system might be hacked through all of these concealed areas. Furthermore, a person must be assigned to watch the camera on a regular basis, and if he fails to do so, a variety of issues may occur...

As a result, a mobile robot might be used to address these issues. A robot might traverse the surveillance areas on its own, making personal judgements when it observes possibly lethal behaviors or activities and then taking action, such as issuing warnings. Object tracking is a video editing method that keeps track of where moving objects are in a film. This may be done by detecting and monitoring the property of a moving item, such as its color. After that, the technique may be used to track the object's movement over time.. The most well-known color tracking systems track a certain color feature. However, when the camera moves, the detected color attribute may disappear as the environment changes. In this case, tracking may be utilized to locate a missing item. As a result, new methods for establishing the color function depending on the working environment of the camera are required.

\section{LITERATURE REVIEW}

Users have recently begun to establish large-scale camera networking as a result of the emergence of low-cost lenses. This increasing number of cameras might open the way for new signal conditioning techniques that use a large number of sensors dispersed across a large area. Object tracking is a novel method of recognizing moving things in video sequences by following them with the camera continually. Its main goal is to match the target items' shape or attributes in successive video sequences.

Shen et al. suggested a new hierarchical spatial saliencybased moving targeting method (2013). To improve detection results, data on temporal and geographical output was also utilized. Our approach correctly and effectively recognizes moving objects in aerial imagery, according to the findings of this test. Furthermore, unlike the HMI technique, this solution has no time delay. This technique, on the other hand, classified item placements as false, unavoidable alarms in all video frames...

To track items, Guo et al. (2012) suggested using an object identification approach in video frames. The simulation results indicate that this method for determining generic class labels is efficient, exact, and long-lasting. Second, enhancing the accuracy of real-time object recognition categorization must be a top focus...

A text recognition technique based on video frame statistics and texture analysis was suggested by Ben Ayed et al (2015). Ben Ayed and his associates are a collection of people who wish to make a difference in the world. The films are divided into separate segments of differing sizes and analyzed using the har wavelet method. Furthermore, a neural network was used to classify text and non-text elements. The study's major focus should, however, be on removing loud places from the surroundings and removing sites such as texts..

Viswanath et al. recommended using panoramic backdrop modelling (2015). This approach was utilized to represent the entire visual element in Gaussian space-time. Moving chemicals may be recognized with fewer false alarms, according to simulation studies. This strategy, however, fails when the section's required capability is not available... Soundrapandiyan and Mouli were provided a unique approach for adjusting to pedestrian detection (2015). Furthermore, the pixel intensities in the image distinguished the foreground from the distance. To emphasize the front borders, they used a high boost filter. The findings of the subjective appraisal and evaluation demonstrate that the proposed technique is successful, with a high detection rate of about $90 \%$ in the pedestrian, when compared to other existing single picture methodologies. They hoped to improve the method's efficacy in the future by boosting detection control and reducing false positives, according to sequence image techniques. 
Based on a relationship between blocks in the current and background pictures, Ramya and Rajeswari created an updated frame difference technique that recognizes pixels as depth of field. The blocks in the current image that are closely related to the backdrop image are referred to as the background. Based on the pixel-based evaluation, the third block is categorized as either front or bottom. According to these experiments, the frame difference approach increases, especially when accuracy and speed are combined. However, this study needs focus on extra information available on blocks, such as shape and edge, in order to improve detection accuracy.

Based on a relationship between blocks in the current and background pictures, Ramya and Rajeswari created an updated frame difference technique that recognizes pixels as depth of field. The blocks in the current image that are closely related to the backing image are referred to as the background. Based on the pixel-based evaluation, the third block is categorized as either front or bottom. According to these experiments, the frame difference approach increases, especially when accuracy and speed are combined. However, in order to improve detection accuracy, this study needs concentrate on other information available on blocks, such as shape and edge.

\section{OBJECTIVES}

The goal of the project is to keep an eye on the object. In response, an item's HSV value is calculated, and the same object is then monitored using the HSV values that have previously been generated. We employ two models in our work. One is used to calculate an item's HSV value, while the other is used to maintain track of it. OpenCV is used to maintain track of the object...

\section{METHODOLOGY}

There are two steps to the approach that I use in my work.

\section{A. HSV value detection:}

This is done in real time by positioning the object in front of another sensor while the model is running. When the code is run, the image of the object appears on the screen. A colorful image emerges on the screen. After converting this RGB image to an HSV image, the values must be determined. To assist with this, we've created a Graphical User Interface (GUI) paradigm. The six values that must be found are hue $(\min )$, hue $(\max )$, saturation $(\min )$, saturation $(\max )$, value $(\min )$, and value $(\max )(\max )$. The usage of a graphical user interface simplifies the process of locating these numbers (GUI). The vertical tabs for all six values must be adjusted to the point where the tracking item turns white and the surrounding area turns black. This method is used to determine the object's HSV value, which is subsequently utilized in the tracking stage that followed. The six matching outcomes are all in the 0 to 255 range. The first step is to select a research area (ROI). There are four steps to it.:
- Background subtraction

- Noise elimination

- Object tracking

- Behaviour analysis

1). Background Subtraction: To remove the foreground, the live RGB recordings are first converted to grayscale images. Because grey photos process slower than colour photographs, they have been employed as input variables for the clipping approach $[3,4]$. The IABMM[6] algorithm then uses white pixels to identify motion parallax as forefront items and displays them in a binary image, while black pixels are used to assign all static objects to backgrounds.

2). Noise Elimination: During noise removal, noise reduction is employed to eliminate any noises produced by reflexions or motion flushes. Median filtering and binary structural approaches are used to eliminate noise. While maintaining significant data, the midway filter[6] eliminates and restores "salt-andpepper" noise. Due to noise caused by a shifting backdrop or lighting environment, foreground features may be misread and classified as background pixels, resulting in gaps or difficulty. In morphological processes, dilatation and erosion[7] are used to reduce noise by connecting likely front-end locations and removing any that are incorrect... The picture pixel is swapped with the maximum value below the anchor after calculating the reference implementation's highest pixel value. Degradation is also an inverted function, which means it operates with the lowest numbers rather than the greatest. Narrowing and erosion are used to produce morphological closeness, causing bright spots to become blobs and increasing foreground detection (represented by white blobs) refer to Fig 2.

3). Object Tracking: The LTCLA algorithm[8], a rapid labelling technique that simultaneously recognises connected pieces and their outlines, is used to monitor the (if any) splodge of the resultant binary loud sound image, which is tracked using the object-tracking approach.

A crucial part of this methodology is a silhouette tracing method that uses a tracer to identify the exterior and interior contours of each element. When an outline point is found, the tracer searches its eight neighbours in a clockwise fashion for more pattern points.

The choice of which blob to follow is the next step in the object tracking stage. The monitoring object is identified as the largest moving blob found in this investigation. Despite the fact that the technique may monitor a large number of targets, the number of observed items has been shown to have a significant impact on the method's effectiveness. This method just chooses and follows the largest blob...

4). Behaviour Analysis: After selecting the largest blob, the ROI method's final stage is to analyse the blob's behaviour. It is possible to identify the item's area, 
centre, and speed, as well as its observable traits. In computing these behaviours in words, a ROI is created by a bounding box that encompasses the target item and is given with the item's greatest width and height. The area of an item is approximated by counting the number of pixels in the tracked blob.

The colour filter will analyse both the ROI and the entire colour space of the picture after the ROI has been located.

HSV is chosen over RGB because it is better at distinguishing things like shadows, shades, and highlights

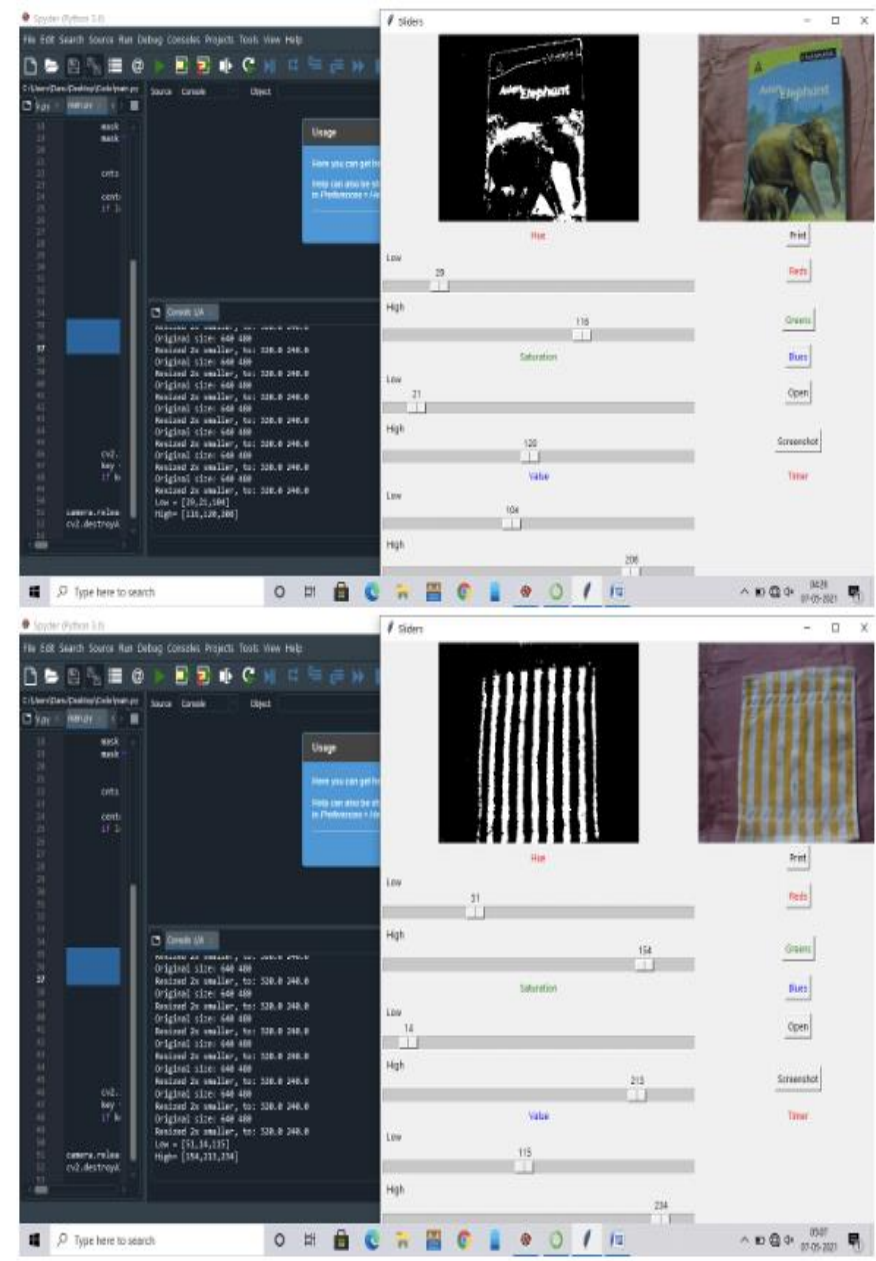

in a variety of lighting circumstances. As a result, there are fewer segments in the filter than in $\operatorname{RGB}[9,10]$. Furthermore, the RGB color space combines neighboring color items of different colors, resulting in bland results, whereas the HSV color space separates the intensity from the color data, resulting in a result that differentiates between both areas bordering color objects of different colors by sharing the limits and maintaining high degree of precision for each pixel [11]. The color filter supplied was created to cover the whole HSV color range. The quantity in HSV space can be affected by system restrictions such as camera resolution and algorithm processor performance.

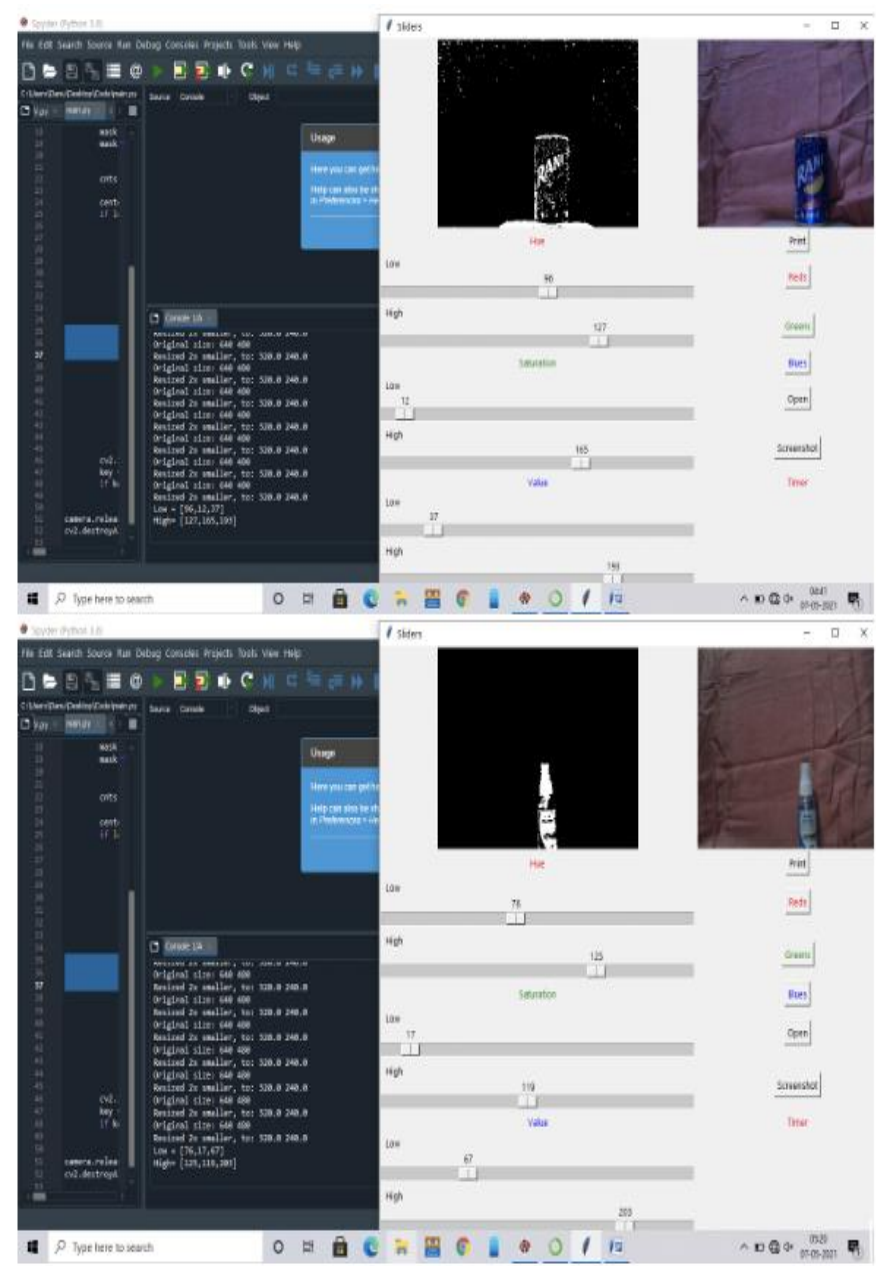

Fig 2: Real Time Gesture Recognition

\section{B. Tracking the Object:}

The HSV computed values from the first stage are utilized to track the object in the second model. For this, we utilize the OpenCV package... 


\section{RESULTS AND OBSERVATIONS}
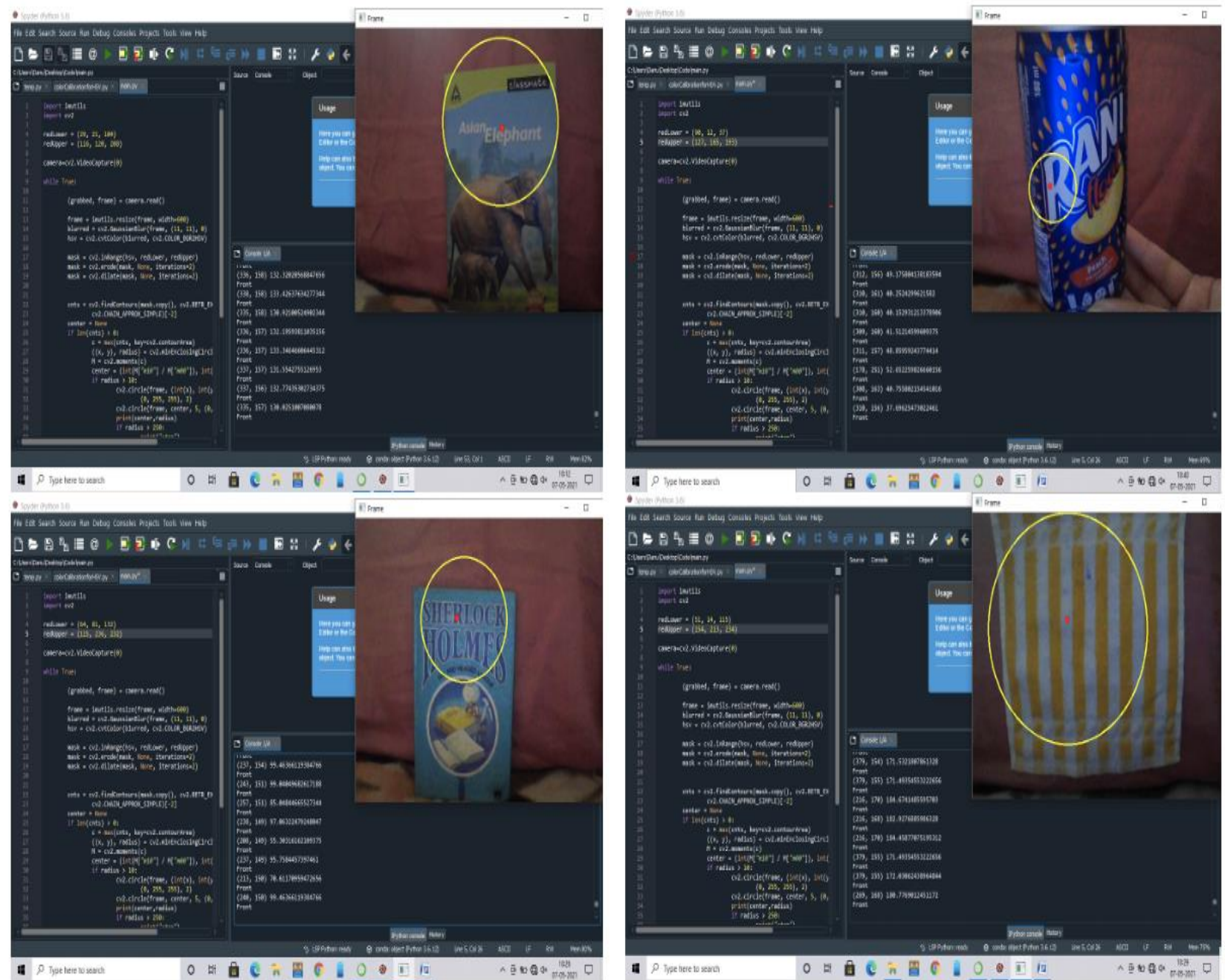

Fig 3: Real Time Object Tracking

The OpenCV library is used to track the entities. The model is ringed by the tracked item as shown in Fig 3, which is positioned in front of the camera. The validity of this approach was found to be $90 \%$.

\section{CONCLUSION}

The HSV value of an object was determined in this experiment, and the image was observed. It was discovered that tracking an item using the HSV value is extremely precise and seldom fails. This strategy might be applied in a variety of disciplines, including defense..

\section{FUTURE WORK}

Although the system can recognise gestures successfully, some extension is still possible. For example, by applying knowledge driven methodology such as Belief Rule Base (BRB), which is widely used where uncertainty becomes an issue [17] [18] [19] [20] [21]. Hence, the recognition of gesture can be accomplished more accurately. More gestures can be added to list of recognition. It was assumed that the background should be less complex. Therefore, recognition of gestures in complex background can be another extension. Recognition of gestures made with both hands is not possible by this system. Therefore, another future work can be the recognition of gestures made with both hands.

\section{REFERENCES}

[1] Aggarwal, A., Biswas, S., Singh, S., Sural, S. \& Majumdar, A.K., 2006. Object Tracking Using Background Subtraction and Motion Estimation in MPEG Videos, in 7th Asian Conference on Computer Vision. SpringerVerlag Berlin Heidelberg, pp. 121-130. doi:10.1007/11612704_13

[2] Aldhaheri, A.R. \& Edirisinghe, E.A., 2014. Detection and Classification of a Moving Object in a Video Stream, in: Proc. of the Intl. Conf. on Advances in Computing and Information Technology. Institute of Research Engineers and Doctors, Saudi Arabia, pp. 105-111. doi:10.3850/ 978-98107-8859-9_23 760 Mukesh Tiwari and Dr. Rakesh Singhai

[3] Ali, S.S. \& Zafar, M.F., 2009. A robust adaptive method for detection and tracking of moving objects, in: 2009 
International Conference on Emerging Technologies. IEEE, pp. 262-266. doi:10.1109/ICET.2009.5353164

[4] Amandeep, Goyal, M., 2015. Review: Moving Object Detection Techniques. Int. J. Comput. Sci. Mob. Comput. 4, $345-349$.

[5] Athanesious, J. \& Suresh, P., 2012. Systematic Survey on Object Tracking Methods in Video. Int. J. Adv. Res. Comput. Eng. Technol. 1, 242-247.

[6] Avidan, S., 2004. Support vector is tracking. IEEE Trans. Pattern Anal. Mach. Intell. 26, 1064-1072. doi:10.1109/TPAMI.2004.53

[7] Badrinarayanan, V., Perez, P., Le Clerc, F. \& Oisel, L., 2007. Probabilistic Color and Adaptive Multi-Feature Tracking with Dynamically Switched Priority Between Cues, in: 2007 IEEE 11th International Conference on Computer Vision. IEEE, pp. 1-8. doi:10.1109/ICCV.2007.4408955

[8] Bagherpour, P., Cheraghi, S.A. \& Bin Mohd Mokji, M., 2012. Upper body tracking using KLT and Kalman filter. Procedia Comput. Sci. 13, 185-191. doi:10.1016/j.procs.2012.09.127

[9] Balasubramanian, A., Kamate, S., \& Yilmazer, N., 2014. Utilization of robust video processing techniques to aid efficient object detection and tracking. Procedia Comput. Sci. 36, 579-586. doi:10.1016/j.procs.2014.09.057

[10] Ben Ayed, A., Ben Halima, M., \& Alimi, A.M., 2015. MapReduce-based text detection in big data natural scene videos. Procedia Comput. Sci. 53, 216- 223. doi:10.1016/j.procs.2015.07.297

[11] Blackman, S.S., 2004. Multiple hypotheses tracking for multiple target tracking. IEEE Aerosp. Electron. Syst. Mag. 19, 5-18. doi:10.1109/MAES.2004.1263228

[12] Chakravarthy, S., Aved, A., Shirvani, S., Annappa, M., \& Blasch, E., 2015. Adapting Stream Processing Framework for Video Analysis. Procedia Comput. Sci. 51, 2648-2657. doi:10.1016/j.procs.2015.05.372

[13] Chandrajit, M., Girisha, R., \& Vasudev, T., 2016. Multiple Objects Tracking in Surveillance Video Using Color and $\mathrm{Hu}$ Moments. Signal Image Process. An Int. J. 7, 15-27. doi:10.5121/sipij.2016.7302

[14] Chate, M., Amudha, S., \& Gohokar, V., 2012. Object Detection and tracking in Video Sequences. ACEEE Int. J. Signal Image Process. 3.

[15] L. Bottou, "Large-scale machine learning with stochastic gradient de-scent," in Proceedings of COMPSTAT'2010. Springer, 2010, pp 177-186

[16] L. Perez and J. Wang, "The effectiveness of data augmentation in image classification using deeplearning," arXiv preprint arXiv:1712.04621, 2017.

[17] R. U. Islam, M. S. Hossain, and K. Andersson. "A novel anomalv detection algorithm for sensor data under uncertainty," Soft Computing, vol. 22, no. 5, pp. 1623-1639, 2018.

[18] M. S. Hossain, S. Rahaman, A.-L. Kor, K. Andersson, and C. Pattinson. "A belief rule based expert system for datacenter pue prediction under uncertainty," IEEE Transactions on Sustainable Computing, vol. 2 no. 2. pp. 140-153, 2017.

[19] M. S. Hossain, F. Ahmed, K. Andersson et al., "A belief rule Based expert system to assess tuberculosis under uncertainty," Journal of medical systems, vol. 41, no. 3, p. 43, 2017.

[20] M. S. Hossain, P.-O. Zander, M. S. Kamal, and L. Chowdhury, "Belief- rule-based expert systems for evaluation of -government: a case study," Expert Systems, vol. 32, no. 5, pp. 563-577, 2015.

[21] R.Ul Islam, K. Anderson, and M. S. Hossain, "A web based Belief rule based expert system to predict flood," in Proceedings of the 17th International conference on information integration and web-based applications \& services. ACM, 2015, p. 